\title{
Genetic analysis of multiple sclerosis in Shetland
}

\author{
D F ROBERTS, ${ }^{1}$ M J ROBERTS, ${ }^{1}$ AND D C POSKANZER ${ }^{2}$ \\ From the Department of Human Genetics, ${ }^{1}$ University of Newcastle upon Tyne, UK, and the Department of \\ Neurology, ${ }^{2}$ Massachusetts General Hospital, Boston, USA
}

SUMMARY In a family study of all patients with multiple sclerosis in Shetland the number of inbred patients, although high for Britain and higher than in Orkney, is not higher than the number among controls, and the inbreeding coefficients suggest that there is no recessive involvement of rare genes in the aetiology. The kinship coefficients show close interweaving of ancestries of patients and controls and eliminate from the aetiology any involvement of recently introduced single genes dominant or codominant in effect. Family histories show that single locus inheritance is unlikely. Any genetic involvement is likely to be polygenic, but on a multifactorial hypothesis the estimates of heritability are very low. The findings suggest that the genetic contribution to the aetiology of the disease in Shetland is slight.

In a recent study of multiple sclerosis in Orkney ${ }^{1}$ analysis of family data suggested that the genetic contribution to the aetiology of the disease in Orkney is not high. Rare genes of recessive, dominant, or codominant effect did not appear to be involved, and single locus inheritance was considered unlikely. On a multifactorial hypothesis, heritability estimates were only moderate. It was clearly desirable to attempt to confirm these findings by similar analyses in other populations, and a study in the Shetland Islands is the subject of the present report.

As with the Orcadians, the population of the Shetland Islands has several features making it particularly suitable for such an inquiry. The prevalence of multiple sclerosis is high, the area is geographically well defined, the vital records are extensive and accurate, and there is a strong sense of identity in the population which has been relatively constant, for although there has been a steady flow of emigrants, relatively few immigrants in the past few decades have settled and contributed to the genepool of the population, certainly not until the recent oil exploitation.

\section{Material and methods}

All patients with multiple sclerosis in the Shetland Islands alive on 1 December 1974 were identified. There were 33,28 of whom were diagnosed as probable cases and five as possible; 31 had been born in Shetland and two elsewhere. For each Shetland born patient a contiguous control was selected who was not a member of the same immediate family, who was born in the same parish in the same year, was of the same sex, had resided in the same area for the first 15 years of life, and was not affected. Where a control was subsequently found to have a neurological disorder, or to be a first or second degree relative of the patient to whom he was matched, a replacement control was obtained. For each Shetland born patient also a discontiguous control was selected, who though of the same sex and born in the same year was born in a parish other than that of the patient. No controls were included for the two patients from outside Shetland.

Each patient and each contiguous control was interviewed to obtain a family history, and where the presence of multiple sclerosis was suspected in a relative, hospital notes and other documentary evidence were sought for verification. The stated details of each propositus and control were verified from documentary sources, and ancestry was then traced back through vital records, parish records, electoral returns, land deeds, and the wide variety of documentation that is available for this type of genetic investigation. In all, some 45000 record searches were completed relating to some 13000 individuals back to an arbitrary baseline taken as 1775. From these data the intricate web of relationships was established, and inbreeding and kinship were measured by the appropriate coefficients ( $\mathrm{F}$ and $\emptyset$ respectively). 


\section{Results}

\section{CONSANGUINITY}

The examination of inbreeding in the Shetland samples showed some features similar to, and others different from, those in Orkney. Out of 31 Shetland born patients, five $(16 \cdot 1 \%)$ were the product of consanguineous unions - that is, were inbred to some extent. Of these, two were from first cousin unions. This proportion is considerably higher than that from the indirect estimates of consanguinity available for populations elsewhere in the British Isles and slightly higher than in Orkney.

In the Shetland control series, however, similar figures emerged. Among the 31 contiguous controls there are eight inbred individuals $(25.8 \%)$, and among the 31 discontiguous controls six (19.4\%). It appears therefore that the raised incidence of inbred individuals observed in patients with multiple sclerosis in Shetland is a feature of the population as a whole rather than of any association with the disease.

The inbreeding coefficients show a similar rise (table 1). Among the 31 patients, the mean inbreeding coefficient is 0.00592 , in the contiguous controls 0.00599 , and in the discontiguous controls 0.00302 . In those individuals who are inbred the mean in patients $(0.03672)$ is higher than in the contiguous controls (mean 0.02319) and the discontiguous (mean 0.01563). Only two of the five inbred patients have inbreeding coefficients under 0.03 , as do 10 of the 14 inbred controls. There is a slight suggestion, therefore, that the distribution of inbreeding coefficients may be shifted slightly upwards in the patients.

These levels are slightly higher than the corresponding figures in Orkney. It seems that as the Shetland population is rather more inbred, but still not highly so, and widespread more severe effects of close inbreeding are not to be expected.

\section{KINSHIP}

Examining the relationship of the 31 Shetland born patients with every other gives 465 possible paired comparisons, and hence 465 kinship coefficients $(\varnothing)$. The number of possible comparisons between every patient and every control is 1922 .

The mean kinship coefficient among all patients is 0.000393 (table 2), the mean among contiguous controls is 0.000169 , and between discontiguous controls 0.000777 . The mean for the affecteds is intermediate between those of the two groups of controls. In the patient comparisons there are 449 zero coefficients and 16 non-zero, the mean of the non-zero coefficients being $0 \cdot 011414$. Among the contiguous controls there are 450 zero and 15 non-zero, figures similar to those in patients, and the mean of the non-zero coefficients is 0.005241 . Among the discontiguous controls, there are 440 zero, 25 non-zero, and the mean non-zero is 0.014453 . The difference in the numbers of positive coefficients in the patient, contiguous control and discontiguous control sets is not significant. Only $10-8$ of the Shetland born patients are not related in some way to at least one of the other patients; 15 of the

Table 1 Inbreeding in Shetland

\begin{tabular}{|c|c|c|c|c|c|c|}
\hline & No of subjects & Mean $F$ & $\begin{array}{l}\text { No with } \\
\text { positive } F\end{array}$ & $\%$ & $\begin{array}{l}\text { No from } \\
\text { Ist cousin unions }\end{array}$ & $\begin{array}{l}\text { Mean of } \\
\text { inbred }\end{array}$ \\
\hline $\begin{array}{l}\text { Patients } \\
\text { Contiguous controls } \\
\text { Discontiguous controls }\end{array}$ & $\begin{array}{l}31 \\
31 \\
31\end{array}$ & $\begin{array}{l}0.00592 \\
0.00599 \\
0.00302\end{array}$ & $\begin{array}{l}5 \\
8 \\
6\end{array}$ & $\begin{array}{l}(16 \cdot 1) \\
(25 \cdot 8) \\
(19 \cdot 4)\end{array}$ & $\begin{array}{l}2 \\
2 \\
-\end{array}$ & $\begin{array}{l}0.03672 \\
0.02319 \\
0.01563\end{array}$ \\
\hline
\end{tabular}

Table 2 Kinship coefficients in Shetland

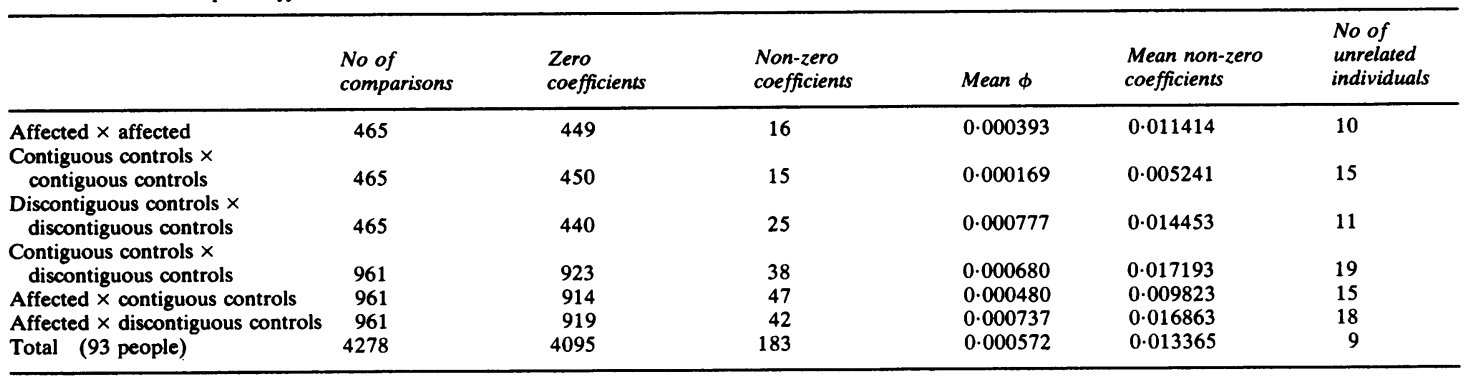


contiguous controls are not related to some other contiguous control; and only 11 discontiguous controls are not related to at least one other discontiguous control. Only 11 of the discontiguous or contiguous controls are unrelated to some other control. There appears to be an intricate network of relationships among patients, and similar networks among both groups of controls, but there is no reason to believe that the patients are more closely related to each other than are the controls.

Of the 961 possible comparisons between all patients and contiguous controls, there are 914 zero coefficients and 47 non-zero, the mean being 0.000480 . The mean of the non-zero coefficients is 0.009823 . Of the 961 possible comparisons between patients and discontiguous controls, there are 919 zero and 42 non-zero coefficients: the mean is 0.000737 , and the mean of the non-zero coefficients is $\mathbf{0 . 0 1 6 8 6 3}$. Again, the proportion of non-zero coefficients is not significantly different from that within patients or either group of controls. Out of the total number of 93 subjects, only nine are not related to at least one other. The intricate network of relationships that exists within the patients and each control group is seen also to unite them. There is therefore no reason to regard patients as being different in their ancestry from the controls, and all three sets of families are seen to be inextricably intertwined together.

\section{FAMILY HISTORIES}

The histories collected in the field survey are remarkably empty of affected relatives. Information was collected regarding 214 first degree, 578 second degree, and 499 third degree relatives of the patients. Of these, cases of multiple sclerosis were reported in only one of the first degree relatives, in two second degree, and in two third degree. These give proportions of one in 214 , one in 289 , and one in 250 respectively. For the contiguous controls, there were 196 first degree relatives, 434 second degree, and 423 third degree, whose histories were recorded. Of these, multiple sclerosis had occurred in one first degree relative (a sister) and one second degree; diagnosis in the former was only established after the survey was completed.

The occurrence in the control series of two affected relatives out of 1053 is close to the population prevalence (1 in 534). The incidence in relatives of patients (5 in 1291) is raised, about double the population prevalence, but is somewhat below those observed in families elsewhere in Britain. In published reports approximately one in $\mathbf{1 7 5}$ parents and one in 100 siblings of patients are affected. If the familial distribution in Shetland were the same as elsewhere in Britain the Shetland patient data would be expected to show two patients among first degree relatives, two to three patients among second degree relatives, and one to two among third degree relatives. Over these degrees, five affected occur instead of six to seven. The number of relatives considered exclude those below the age at which multiple sclerosis is likely to develop, so they can all be regarded as informative. The apparent slight deficit of first degree relatives is possibly due to chance in what are relatively small samples. But there may also have been some underreporting in the field, perhaps deliberately due to withholding of information by the proband or unintentionally due to his lack of knowledge of the diagnosis.

Of the 33 patients' pedigrees, none suggests simple dominant, recessive, or X-linked inheritance. All the affected relatives occur at such a distance from the propositus as to prove that if a dominant or codominant gene is operative, environmental or other genetic factors must be sufficiently important as to prevent its manifestation in almost all of those who carry it; from the information in the present data, penetrance can be estimated at approximately $9 \%$. Again, the pedigree data do not support the hypothesis of a simple recessive disorder, for of the 121 siblings of the patients, none is affected, far too few on this hypothesis. The occurrence of so many affected women ( 19 women and 14 men) eliminates a sex linked recessive mode of inheritance.

Multifactorial inheritance is an alternative to monogenic inheritance, and there are many diseases to which the multifactorial model appears to apply. The extent of the additive genetic contribution to the total variance in liability to develop the condition is estimated by the heritability $\left(h^{2}\right)$. This is normally calculated by comparison of incidences in relatives of different degrees with control series or with population prevalences. The first method is precluded in the present material by the small number of affected relatives of controls. From the population prevalence, however, heritability from first degree relatives may be calculated as $18 \cdot 8 \% \pm$ $19 \%$, a figure quite close to that from second degree relatives $\left(\mathrm{h}^{2}=24 \cdot 2 \% \pm 32 \%\right)$, but in both the standard error is so high as to render these estimates virtually meaningless. Pooling estimates from first, second, and third degree relatives, the weighted heritability is $23 \cdot 0 \% \pm 15 \cdot 8 \%$. These estimates are considerably lower than those calculated from the studies of Curtius, ${ }^{2}$ Pratt et al, ${ }^{3}$ Sutherland, ${ }^{4}$ Schapira et al, ${ }^{5}$ and Millar and Allison ${ }^{6}$ and also lower than the Orkney estimates. ${ }^{1}$ The size of the standard errors indicates that, although the total population of patients was included, the numbers of informative relatives are too few for a reliable estimate of heritability; all that can be said is that the true figure 
is certainly not as high as for conditions such as cleft lip and palate, or analysing spondylitis.

\section{Discussion}

The present findings give information on several genetic hypotheses. In the consanguinity analysis the inbreeding is a phenomenon of the population as a whole, rather than of the patients. The lack of elevation of the number of inbred patients by comparison with the controls, or of any pronounced displacement of the distribution of the inbreeding coefficients, appears to argue against, though not eliminate conclusively, recessive involvement of rare genes. In the kinship analysis the similarity of the mean kinship coefficients and their distribution within patients, within contiguous and within discontiguous controls, and their similarity moreover to those between patients and controls, appears to eliminate involvement of genes of dominant or codominant effect recently introduced in the population. The kinship analysis does not eliminate involvement of such genes widely distributed, long established in the population, but of very low penetrance.

The family histories and inspection of pedigrees suggest that single locus inheritance of multiple sclerosis is unlikely. There is little real evidence for dominance or codominance, such as is implied in recent discussions of a "gene" for multiple sclerosis linked to the histocompatibility complex. If such a gene exists its effects are grossly outweighed by environmental factors, and the kinship analysis indicates that it must have been introduced very early in the history of the population. While the consanguinity analysis does not totally exclude a hypothesis of recessive involvement of rare genes, the family analysis gives no support to this hypothesis, for if it were correct one would expect many more of the siblings of patients to be affected than appears to be the case. Again, however, if such genes recessive in effect exist they must be heavily obscured by environmental factors. Sex linkage also may be eliminated from the sex distribution of the disorder that is in the opposite direction to that required on this hypothesis. The pedigrees themselves appear much more compatible with the alternative, polygenic hypothesis that is suggested by the data from the Orkney and other studies. ${ }^{9}$ The proportions of affected relatives of different degrees are far too low in the Shetland material for this hypothesis to be examined properly, but certainly the family findings do not contradict it.

Interpretation of the reported family histories in genetic analysis depends on the accuracy of the diagnosis in those regarded as affected relatives. In field surveys some informants give the impression that any relative with a suspicion of a disorder is affected, and so their evidence overestimates the number, while others declare no knowledge of any other case in the family, either deliberately through a concious suppression of information, but more usually unconsciously through lack of the necessary knowledge. This sort of difficulty necessitates the use of documentary evidence as well as that of the field survey, but this may be lacking in a proportion of cases which it will therefore be impossible to assign one way or other. In the present study, where it was not possible to obtain confirmation, the view of the field worker, a general practitioner with long experience in the islands and personal knowledge of many of the families included, was accepted. If there is a bias it seems to lie in the direction of underreporting.

Nevertheless, the study as a whole, an analysis of multiple sclerosis in a total relatively compact population, applying modern methods of population genetics to an aetiological problem, has produced valuable results. It indicates that any genetic component that there may be in multiple sclerosis is not simple. Monogenic inheritance in any form appears unlikely, unless there is greatly reduced penetrance and gross environmental interference. The results are not compatible with a more complex aetiology, in which the genetic contribution is polygenic, and the heritability estimates suggest that it is relatively slight by comparison with non-genetico elements in the aetiology, although the precises estimates are somewhat unsatisfactory. Both these main findings appear to support those from other recent studies. ${ }^{178}$

Acknowledgment is gratefully made to Dr Mary Mack of Shetland for her invaluable help in the field: to Mrs J A Cowie and her associates for tracing the family histories; and to the staff of Register House, Edinburgh. This study was supported by the National Institute of Neurological and Communicative Disorders and Stroke (contracts NIH-NINCDS N01-NS-4-2321 and N01-NS-6-2337) and the Multiple Sclerosic Society of Great Britain and Northern Ireland.

\section{References}

${ }^{1}$ Roberts DF, Roberts MJ, Poskanzer DC. Genetic analysis of multiple sclerosis in Orkney.J Epidemiol Community Health 1979; 33: 229-35.

${ }^{2}$ Curtius F. Multiple Sklerose und Erbanlage. Leipzig: Thieme, 1933.

${ }^{3}$ Pratt RTC, Compston ND, McAlpine D. The familial incidence of disseminated sclerosis and its significance. Brain 1951; 74: 191-232. 
${ }^{4}$ Sutherland JM. Observations on the prevalence of multiple sclerosis in Northern Scotland. Brain 1956; 79: 635-54.

${ }^{5}$ Schapira K, Poskanzer DC, Miller H. Familial and conjugal multiple sclerosis. Brain $1963 ; 86 ; 315-32$.

${ }^{6}$ Millar JHD, Allison RS. Familial incidence of disseminated sclerosis in Northern Ireland. Ulster Med J 1954; 23: 29-91.

${ }^{7}$ Poskanzer DC, Sheridan JL, Prenney LB, Walker AM. Multiple sclerosis in the Orkney and Shetland Islands. II. Search for an exogenous aetiology. $J$ Epidemiol Community Health 1980; 34: 240-52.
${ }^{8}$ Poskanzer DC, Walker AM, Prenney LB, Sheridan JL. The aetiology of multiple sclerosis: temporal spatial clustering indicating two environmental exposures before onset. Neurology 1981; 31: 708-13.

${ }^{9}$ Roberts DF, Bates D. The genetic contribution to multiple sclerosis: evidence from North-East England. J Neurol Sci 1982; 54: 287-93. 